\title{
A model of endogenous political party platforms *
}

\author{
Andrei M. Gomberg ${ }^{1}$, Francisco Marhuenda ${ }^{2}$, and Ignacio Ortuño-Ortín ${ }^{3}$ \\ 1 Department of Economics and CIE, Instituto Tecnológico Autónomo de México, México \\ D.F. 10700, MEXICO \\ 2 Department of Economics, Universidad Carlos III, 28903 Getafe (Madrid), SPAIN \\ 3 Department of Economics and IVIE, University of Alicante, Alicante, SPAIN \\ (e-mail: ortin@merlin.fae.ua.es)
}

Received: November 20, 2002; revised version: August 21, 2003

Summary. We develop a model of endogenous party platform formation in a multidimensional policy space. Party platforms depend on the composition of the parties' primary electorate. The overall social outcome is taken to be a weighted average of party platforms and individuals vote strategically. Equilibrium is defined to obtain when no group of voters can shift the social outcome in its favor by deviating and the party platforms are consistent with their electorate. We provide sufficient conditions for existence of equilibria.

Keywords and Phrases: Endogenous party formation, Multidimensional policy space.

JEL Classification Numbers: D72, C62.

\section{Introduction}

In the extensive literature on political economy and party competition it is commonly assumed that there exists a policy space in which the platforms proposed by the parties can be seen as points. Voters have well-defined preferences over such a space and, given an electoral rule, vote, possibly in a strategic manner. Many

* We thank A. Caplin, S. Chattopadhyay, C. Martinelli and J. D. Moreno-Ternero and two anonymous referees for helpful comments. This research started while Ortuño-Ortín was a visitor in the Department of Economics at NYU; he thanks for the kind hospitality of this institution. F. Marhuenda and I. OrtuñoOrtín gratefully acknowledge financial support from the Spanish Ministry of Science and Technology Project BEC2001-1653 and Project BEC2001-0980, respectively; A. Gomberg gratefully acknowledges the financial support from the Asociación Mexicana de Cultura. 
papers, dating back to Wittman [25], assume that parties are "ideological", that is, that they have preferences over the policy space. In this interpretation, one may view parties as institutions that represent contesting interest groups in the society. An ideological party adopts a platform that maximizes its expected utility, subject to electability considerations. ${ }^{1}$ Most of the papers in the Wittman tradition share the same basic assumption: parties, and their ideology, are exogenously given.

The ideology of a party, however, can hardly be viewed as an intrinsic feature of the party itself, since it would generally depend on the preferences of its members. At the same time, individuals choose to join a party taking into account its stand on issues. It, therefore, seems that one should consider party membership and party ideology simultaneously, so that both party membership and their ideologies are endogenized.

In this paper, we provide a model of endogenous party formation in a setting with a multi-dimensional policy space. The basic idea of our model is the following. A large society has to implement a vector of policies via a democratic procedure. There are two established political parties. The parties function as aggregators of preferences of the population and they present well-defined platforms for the general vote. Agents are seen in a twofold role, both as voters and as party members. Each agent can belong to (at most) one party. Preferences (ideology) of each party are determined, according to some fixed aggregation rule, by the preferences of its members. Preferences of parties will determine their electoral platforms. In the general election, agents vote taking party platforms as fixed. After the vote takes place, society implements a policy based on its results.

It may actually happen, that some agents prefer to vote for a party different from the one they belong to. We view such a situation as unstable and inconsistent with the system being in the overall equilibrium. Indeed, consider, for instance, the longterm political realignment that has been occurring in the United States. Although, it was once rather typical to find "liberal" Republicans and "conservative" Democrats in the formerly "one-party" South, this is becoming increasingly uncommon. The party ideologies having shifted, both types are no longer attracted to their historic political homes even for the purpose of party registration. ${ }^{2}$

Of course, such a shift in party membership, in turn, must imply a change in the preferences of the parties and, as a consequence, a change in their platforms. An overall equilibrium requires that it is in the interest of each party member to vote for the proposal of his or her party. In this way, both the membership of each party and its ideology are endogenously obtained as a function of the overall distribution of voters' preferences. The basic idea here is the same as the one used by Baron [6], Ortuño-Ortín and Roemer [17] and Roemer [24], and it is inspired by the "voting with one's feet" models (see Caplin and Nalebuff [9]). A similar strand of literature,

\footnotetext{
1 A recent and very complete version of such a model is provided in Roemer [24] (see also Osborne [18] for a survey).

2 Admittedly, this shift hasn't been instantaneous. The delay can be best explained by phenomena not modeled in this paper, such as the hierarchical nature of the political system, with different party identities at the local (as distinct from the national) political level, party loyalty, or costly affiliation change.
} 
which studies political party activism with endogenous political party platforms, is represented by Aldrich [1], Gerber and Ortuño-Ortín [11] and Poutvaara [21].

The major features that distinguish the model are the following.

i) The number of political parties is fixed, but ideologies and platforms of parties are not.

ii) Parties aggregate preferences of their members, but do not necessarily behave strategically in their choice of platforms.

iii) The implemented policy is a weighted average of the party proposals.

iv) Agents vote in a strategic way and are allowed to form coalitions.

Only the first two of these features are essential for our results. The first point means that we take an institutional view of political parties. Our parties are not short-term amalgamations of voters and candidates, but semi-permanent organizations that are part of the society's political structure. This reflects the prohibitive cost that non-party and new-party candidates often face in terms of such things as name-recognition, organization, ballot-access, etc. ${ }^{3}$ Emergence of new major parties, which in many established democracies such as the U.S. and U.K. is an extremely rare, once-in-a-few-generations event of almost semi-constitutional impact, is, therefore, not studied here.

We also assume that parties simply aggregate their members' preferences. We, thus, do not allow parties to respond strategically to platforms proposed by each other. This can be justified by considering the case in which parties cannot commit to policies. In this view, the preferences of parties and their leaders are well-known to the society-at-large and altering the platform to achieve success in the general election is not credible. Consequently, the proposals, or platforms, must coincide with the ideal policies of the parties (see also Alesina [2] and Alesina and Rosenthal [3] for this assumption). This is also the assumption of the citizen-candidate model of Osborne and Slivinski [19], Osborne and Tourky [20] and Besley and Coate [7] (in these models candidates play a role similar to parties in our setting).

The formal extension of the model to the strategic setting is, actually, not difficult. Allowing for strategic behavior by parties in their choice of platforms would, however, generate serious difficulties with equilibrium existence. Indeed, the problem of (Nash) equilibrium existence in multi-party spatial games has been well-known for a long time and is particularly hard when the policy space is multidimensional. ${ }^{4}$ Resolving this problem would require imposing very restrictive assumptions on the distribution of individual preferences and/or possible platforms. However, these existence problems have nothing to do with endogenizing party ideology. Rather than concentrating on this well-known difficulty, we want to restrict our attention to the very distinct problem at hand. Of course, in many cases where the game studied is known to have a Nash equilibrium, our results can be easily extended to the strategic setting.

${ }^{3}$ Indeed, even candidates with significant personal following and major ideological disagreements with all existing parties, such as Jesse Jackson in the U.S. Democratic party, or Pat Buchanan (first Republican, later Reform parties), seem to prefer working through established political organizations.

${ }^{4}$ For a general view of the problem see, for example, Roemer [24]. See also Roemer [23] for existence of an alternative equilibrium concept in the two-dimensional case. 
The other assumptions noted above are easily relaxable. In particular, one can easily extend our model to the case in which agents vote sincerely, i.e. agents vote for the platform they like the best according to their preferences, or to the case when the implemented policy coincides with the platform of the winning party (the "winner-takes-all" system). ${ }^{5}$

As noted above, attempts to endogenize party ideology have been undertaken for some time. D. P. Baron [6] considers a multidimensional model with strategic party behavior and sincere voting. Existence of equilibrium, however, is only provided for a very specific two-dimensional example, with three parties and a uniform distribution of voters. I. Ortuño-Ortín and J. E. Roemer [17] consider a specific example of endogenous party formation in which the policy space is onedimensional. J. E. Roemer [24] deals with a two-dimensional policy space problem but the nature of the political parties and the equilibrium concept are different from those standard in the literature.

Our model differs from [6,17] and [24] in several important respects. First, we provide general results on the existence of equilibrium, whereas [6] and [24] provide only specific examples in a two-dimensional model. Furthermore, unlike the example provided in [17], we don't restrict ourselves to the case of a onedimensional policy space. In fact, proving existence of equilibrium in the onedimensional case is relatively easy. For higher dimensions, however, a different approach to establishing equilibrium existence is needed. While the resultant proof is quite involved, it provides important new insights in the nature of political parties. Moreover, the existence result is robust with respect to changes in the party's policy choices.

An important aspect of the paper, is that it clarifies the relationship between the dimensionality of the policy space and the existence of equilibrium. This relationship is often seen as a negative one: the higher the dimension of the policy space is, the harder it is to guarantee existence of equilibrium. This view is a consequence of the well known results in the classical models of political competition, where existence of equilibrium is very rare in two and higher dimensions (an exception is the model in [23]). We show, however, that when ideology and membership of the parties are endogenous to the model, it is harder to obtain existence in the twodimensional case than in the three-dimensional one (This even-odd phenomenon applies to any dimension. Although it is very unusual to assume policy spaces of dimension four or higher). This, however, does not imply that existence of equilibrium in the two-dimensional policy space is always impossible and we indeed show existence for the particular, but important, case in which the ideology of the party coincides with the mean ideology of its members. In the end, one message of the paper is that the odd-even dimensionality of the policy space might play an important role for the equilibrium properties of a multi-party political system. This apparently paradoxical result recalls the one first established by Caplin and Nalebuff[9].

\footnotetext{
5 In this case, it is possible to introduce without any difficulty uncertainty of election outcome into the model. The results would remain the same as in the certainty case.
} 
As noted above, an important feature of our model is the way the overall policy outcome is determined. We assume that the implemented platform does not need to coincide with the platform of the winning party. Namely, the implemented policy is taken to be some convex combination of the parties' platforms. The weights in this combination are assumed to be an increasing function in the vote share (see Grossman and Helpman [13], Ortuño-Ortín [16], Gerber and Ortuño-Ortín [11], Alesina and Rosenthal $[3,4]$ ). We believe that this is a realistic assumption, which captures the way many democratic societies adopt policies.

Another distinctive feature of this paper is our analysis of coalition formation by voters. In our model, voters can form such coalitions; consequently, in equilibrium profitable deviations by coalitions are not allowed (a similar assumption also appears in Alesina and Rosenthal [3,4] and Gerber and Ortuño-Ortín [11]). Other variants of this assumption would yield the same results.

Our research is related to that of Osborne and Slivinski [19] and Besley and Coate [7], who provide a model of "citizen-candidates" in which candidates are endogenously determined. The major difference in our approaches is that the "citizencandidate" literature, for the most part, takes the individual candidates to exist independently, outside any political party structure, which seems to be at odds with the practice of most democratic polities. However, a recent extension of the citizen-candidate model by Riviere [22] introduces political parties as one-shot "cost-sharing organizations" formed, essentially, to finance the candidacy of its leader. This is very much like the leader-driven political organizations in the emerging multi-party democracies, such as Russia in the early 1990's. With the political spectrum restricted to a small discrete set along a single dimension (for the most part, only three positions are allowed: "left - center - right"), these "cost-sharing parties" are endogenized by Riviere [22] within the context of a single election. Our model is different in that we allow for a full multi-dimensional spectrum of ideologies, and take a more institutional view of political organizations as features of society's political system.

The organization of the rest of the paper is as follows. Section 2 sets up the basic model. Section 3 discusses the outcomes of voting games obtained by fixing party platforms. Section 4 provides a two-party equilibrium existence result for the case when the number of policy dimensions is odd. Section 5 analyzes the case of two policy dimensions under the additional assumption that parties choose policies by a mean voter rule.

\section{The model}

Consider a society consisting of a continuum of heterogeneous individuals, with the set of possible types denoted by $A \subset \mathbb{R}^{n}$. The set of agents shall be represented by a measure space $(A, \mathcal{B}, F)$, where $\mathcal{B}$ is the $\sigma$-algebra of (Borel) subsets of $A$ and $F$ is a measure over the type space. We shall assume that $F$ is finite (and, therefore, we may normalize $\int_{A} d F=1$ ), with compact support and hyperdiffuse (that is, every $(n-1)$ dimensional hyperplane in $A$ is of zero measure). 
As it is standard in the theory of non-atomic games, measurable subsets $B \in \mathcal{B}$ of the type space $A$ shall be called coalitions. If for some coalition $C \in \mathcal{B}, F(C)=$ 0 , it shall be called a null coalition.

Let there be a fixed number of political parties ${ }^{6} M=\{1,2, \ldots m\}$. Individuals are free to join any of the parties, resulting in a population partition. Strictly speaking, a partition is a collection of measures $\left\{F_{j}\right\}_{j \in M}$ over $A$ such that for any $E \in \mathcal{B}$, one has $\sum_{j=1}^{m} F_{j}(E)=F(E)$. However, we shall soon impose assumptions which will insure that individuals of the same type will always be strictly better off by going to the same community (except for, possibly, a null coalition of agents), and consequently the $F_{j}$ 's are mutually singular. This suggests that it may be convenient to restrict our attention to population partitions $C=\left\{C_{j}\right\}_{j=1}^{m}$, with $C_{j} \in \mathcal{B}$, that are also partitions of the type space $A$ into $m$ coalitions. The set of all such partitions we shall denote as $\Sigma$. Given such a partition $C \in \Sigma$, the membership share of the party $j \in M$ is $w^{j}=w^{j}\left(C_{j}\right)=F\left(C_{j}\right)$. Hence, the vector of party weights $w=\left(w^{1}, \ldots, w^{m}\right)$ is an element of the $(m-1)$-dimensional simplex $\Delta^{m-1}$.

The society has to implement a vector of policies $x \in X$, where $X$ is a nonempty compact and convex subset of $\mathbb{R}^{n}$. Every individual of type $\alpha \in A$ cares only about overall policy outcomes. For simplicity, we shall restrict the class of individual preferences considered. ${ }^{7}$

Assumption A1: (Euclidean preferences) Individual preferences over $X$ of each agent of type $\alpha \in A$ may be represented by the utility function $u(x ; \alpha)=-\|x-\alpha\|$, where $\|\cdot\|$ stands for the Euclidean norm.

Furthermore, we shall assume that $X$ is sufficiently "large" in the sense that every individual's ideal point is part of the set $X$ of feasible policies. In fact, identifying individuals with their ideal policies, we shall assume:

Assumption A2: $K \subset X$, where $K$ is the convex hull of the support of $F$.

\subsection{Policy outcomes and voting}

Suppose each party $j \in M$ chooses to advocate the policy $p^{j} \in X$. Facing a policy profile $p=\left\{p^{j}\right\}_{j \in M} \in \prod_{j=1}^{m} X \equiv X^{m}$, individuals shall vote, in a manner explained below, inducing some population partition $C$ with corresponding vote shares represented by a vector $w(C) \in \Delta^{m-1}$ (when the underlying partition is clear we shall just write $w$ ).

The overall policy outcome is a function of the manner in which the vote divides between the parties, as well as of which propositions are on offer. In other words,

${ }^{6}$ The set up of the model allows for more than two parties. Our results, however, will be proven for the two-party case.

${ }^{7}$ An extension of the model to accommodate linear preferences, $u(x ; \alpha)=\alpha \cdot x$, as in Gomberg [12] is, in fact, straightforward. 
there is some outcome function $T: X^{m} \times \Delta^{m-1} \rightarrow X$. While, in principle, a general set of outcome functions may be analyzed, we may want to restrict ourselves to special classes of these. In particular, in this paper we shall focus on the "convex combination" (or "weighted average") outcome functions.

Assumption 01: $T(p, w)=\sum_{j=1}^{m} g^{j}(w) p^{j}$, where $g \equiv\left(g^{1}, \ldots g^{m}\right)$ is a continuous function from $\Delta^{m-1}$ to itself.

This assumption entails that the actual policy implemented in the society is a consequence of a political compromise between the competing parties. Of course, we will assume below that the weight each party has in the final outcome is directly linked with the support it obtains.

Notice that for $\varepsilon>0$, arbitrarily small, we can make the function $g$ take the values $g^{j}(w)=1$, for $w^{j}>1 / 2+\varepsilon$. In other words, by choosing the function $g$ appropriately, one can approximate arbitrarily the "winner takes all" situation, i.e., $g^{j}(w)=1$, for $w^{j}>1 / 2$. At any rate, as already mentioned in the introduction, with minor modifications our results can be directly established as well (without having to deal with approximations) for the "winner takes all" case.

The following monotonicity assumption on the outcome function shall be imposed throughout.

Assumption 02: (strict monotonicity) For every $i \in M$, the weight $g^{i}(w)$ assigned to party $i$ is strictly increasing in the vote share $w^{i}$ obtained by that party.

Finally, we assume that, if a party policy proposal attracts no voters, it should have no weight in the final outcome.

Assumption 03: (irrelevance of null voter coalitions) For every party $i \in M$, we have that $g^{i}(w)=0$ whenever $w^{i}=0$.

It follows from $\mathrm{O} 3$ that, for each $i \in M$, if $w^{i}=1$, then $g^{i}(w)=1$. Given a policy profile $p \in X^{m}$ and the outcome function we define the voting game as follows. Each individual votes for one of the political parties. Under the assumption of Euclidean preferences and given the policy proposals represented by $p$ and the voting pattern represented by $w$, the payoff enjoyed by an individual of type $\alpha$ is given by $u(T(p, w) ; \alpha)=-\|\alpha-T(p, w)\|$.

As it is standard in the multi-jurisdictional literature, we have postulated that there is a continuum of voting agents. This is done in order to avoid existence problems resulting from the non-convexity of the individual choice set. However, the continuum assumption has its costs as well and it introduces some technical problems.

In particular, in the context of a model with a continuum of voters, we face the usual problem of voting incentives: since no individual by himself impacts the outcome, any voting behavior may be rationalized. We could have assumed that agents vote sincerely for the party whose policy platform they like the most. However, we are interested in studying implications of some sort of strategic behavior 
on behalf of the voters. ${ }^{8}$ This requires to use one of the equilibrium refinement concepts based on the possibility of deviation by (non-negligible) coalitions. The voting equilibrium concept we employ here is, essentially, the Aumann [5] Strong Nash Equilibrium (SNE), modified to accommodate the model with a continuum of agents. ${ }^{9}$

In general, the problem of existence of SNE in a voting game like the one defined here, is highly non-trivial. Furthermore, even if an SNE exists, it may not be unique. However, for the two-party case it may be shown that, for any policy proposal profile with parties taking distinct policy positions, there does indeed exist a unique SNE of the voting game.

In order to minimize the amount of notation involved it is convenient to express strategy profiles of voters in terms of the partitions of the type space $A$ among parties.

Definition 1. A voting partition $C=\left\{C_{j}\right\}_{j=1}^{m} \in \Sigma$ shall be called a Strong Nash Equilibrium (SNE) of the voting game given by the policy proposal profile $p \in X^{m}$, if there does not exist a partition $C^{\prime}=\left\{C_{j}^{\prime}\right\}_{j=1}^{m} \in \Sigma$ such that for all individuals of types $\alpha \in B=\bigcup_{j=1}^{m} C_{j} \cap\left(A \backslash C_{j}^{\prime}\right) \in \mathcal{B}$,

$$
u\left(T\left(p, w\left(C^{\prime}\right)\right) ; \alpha\right) \geq u(T(p, w(C)) ; \alpha)
$$

and, the set of agents $\alpha \in B$ for which the above inequality is strict has strictly positive measure.

\subsection{Party policy choice}

So far, we have essentially assumed that the policies staked out by the parties are exogenously given. However, party positions naturally depend on preferences of its constituents. We assume that each party possesses a statute, which may be viewed as a mechanism for establishing a policy platform, or program, as a function of the environment. As it has just been discussed, a platform may be viewed as a policy vector $p^{j} \in X$, which the party $j \in M$ would implement, if it could singlehandedly determine the society's policies. It will be generally assumed that a party's platform is always well defined, given some set of relevant data. If party $j \in M$ takes into account just the way in which the population is partitioned, its statute may be viewed as a function $P^{j}: \Sigma \rightarrow X$. Following Caplin and Nalebuff [9], such a

8 The main results of the paper also hold in the case agents vote sincerely for the party whose policy platform they like the most.

${ }^{9}$ SNE may seem to involve much more coalitional reasoning than is likely in a model with a continuum of agents. An alternative would be to consider deviations only by small (but non-null) coalitions of "alike" agents. This could be justified by claiming that similar agents are more likely to be able to communicate in order to be able to behave as a coalition, or by having agents believe that their behavior provides an "example" followed by other individuals with similar preferences. In terms of this model, however, employing this, in principle, less restrictive equilibrium concept has the same effect as employing the SNE. 
party shall be called membership-based. ${ }^{10}$ When all parties are membership-based, we denote the profile of statutes as $P: \Sigma \rightarrow X^{m}$.

Although $P(C)$ takes as an argument the partition of the entire population, the decisions of some people may actually be irrelevant for the party policy choice. In fact, our model does not require that every citizen joins a political party. In this interpretation, we may want to view the overall party membership as the set of "political activists" (possibly, more radical, or just more interested in party politics), in the spirit of Aldrich [1].

A typical example of a membership-based rule would be the median-voter rule, which tells each party to choose the ideal policy of its median member. While this rule is only defined when individuals vary along a single dimension, in a multidimensional context we may study, for example, the mean-voter rule. ${ }^{11}$ In general, any profile of social choice rules aggregating preferences of the members of each party would be in this class.

As noted in the introduction, the parties do not choose their policy platforms in a strategic way. This is a reasonable assumption if, for example, we see $P$ as the function determining the ideal policies of the parties and they cannot make credible commitments to policies. Under this interpretation, once parties are formed, agents can observe their membership and infer the ideal policy that each party will try to implement. Thus, voters will not believe announcements different from $P$ (see Alesina [2])

\subsection{Equilibrium}

In much of the earlier literature, the internal and external politics of the parties have been treated separately. Nevertheless, the two are obviously interrelated, in the sense that party membership determines policy platforms and policy platforms serve to attract citizens to parties. Assuming that party membership coincides with party electorate (an assumption that may be relaxed along the lines discussed in Section 2.2), we say that equilibrium obtains when the voting partition resulting from a policy profile on offer coincides with the membership partition inducing this policy profile.

It is easy to construct equilibria with parties being identical in their policy positions. In this case, any voting pattern corresponds trivially to an SNE of the voting game. Therefore, we are free to chose a population partition to support the identical party positions. Given the apparent "pluralism" of positions on policy issues observed in most political systems, it is, however, of interest to study existence of equilibria with non-identical parties.

10 In principle, more general domains for party statutes may be assumed. In particular, it may be of interest to study the case in which parties respond in some sense to others' actions. An extension of the model along these lines is actually quite straightforward, as suggested by Caplin and Nalebuff [9].

11 This seems particularly fitting in the present context, since, under appropriate conditions, this may be interpreted as the policy proposal that cannot be defeated by a super-majority of party's members in a binary voting (Caplin and Nalebuff [8]). See also Aldrich [1] and Baron [6], where it is assumed that either the party platform or the ideal policy coincides with the mean ideal policy of a party activist. 
Definition 2. Given an outcome function $T$ and a party policy function $P$, we say that $\left(p^{*}, C^{*}\right) \in X^{m} \times \Sigma$ is a multi-party equilibrium if:

(i) $p^{*}=P\left(C^{*}\right)$

(ii) $C^{*}$ is a SNE of the voting game induced by $p^{*}$.

If, furthermore, at least some of the equilibrium party proposals are distinct (i.e., $p^{j *} \neq p^{k *}$ for some $j, k \in M, j \neq k$ ) such equilibrium is called pluralistic.

Given a multi-party equilibrium $\left(p^{*}, C^{*}\right)$, the associated policy outcome is $T^{*}=T\left(p^{*}, w\left(C^{*}\right)\right)$.

\section{Existence of voting equilibrium}

Before tackling the problem of the pluralistic multi-party equilibrium existence, we first have to find conditions which will ensure that the outcome of the voting game obtained when party platforms are fixed is well defined. In fact, it turns out that assumptions $\mathrm{A} 1, \mathrm{O} 1$ and $\mathrm{O} 2$ are sufficient to guarantee existence and uniqueness of SNE in a two-party voting game for a large class of policy profiles. Let $\mathbb{S}^{n-1}=\left\{x=\left(x_{1}, \ldots, x_{n}\right) \in \mathbb{R}^{n}: \sum_{i=1}^{n} x_{i}^{2}=1\right\}$ denote the $(n-1)$-dimensional unit sphere in $\mathbb{R}^{n}$. From now on, we will restrict our attention to the two-party case.

Proposition 1. Let $m=2$ and assume A1, O1 and O2. Then, for each policy profile $p=\left(p_{1}, p_{2}\right) \in X^{2}$ such that $p^{1} \neq p^{2}$, the following holds:

(i) There exists a unique (up to a null coalition of agents) SNE of the voting game;

(ii) In an equilibrium $C=\left\{C_{1}, C_{2}\right\}$, the voters of each party (except, possibly, a zero measure of them) may be separated by a hyperplane through the set of types $A$, i.e. $C_{1}=\{\alpha \in A: \alpha \cdot \pi \geq b\}$ for some $(\pi, b) \in \mathbb{S}^{n-1} \times \mathbb{R}$. Furthermore, $T(P(C), w(C))$ is in the hyperplane $\{\alpha \in A: \alpha \cdot \pi=b\}$, separating $C_{1}$ and $C_{2}$.

From now on, without loss of generality, we include, for convenience, the separating hyperplane $\{\alpha \in A: \alpha \cdot \pi=b\}$ of indifferent individuals in $C_{1}$. To prove the proposition we shall rely on the following lemma.

Lemma 1. Under the assumptions of Proposition 1, a partition $C \in \Sigma$ is a Strong Nash Equilibrium of the voting game if and only if

$$
F\left(C_{j} \cap\left\{\alpha \in A: \alpha \cdot\left(p^{j}-p^{i}\right)<T(p, w(C)) \cdot\left(p^{j}-p^{i}\right)\right\}\right)=0
$$

for any $i, j \in M$ such that $i \neq j$.

Proof of Lemma 1. I. Sufficiency. Fix $i, j \in M$ with $i \neq j$. Let $C \in \Sigma$ be a partition such that the above is true. For each $\alpha \in A$, consider $W_{\alpha}:[0,1] \rightarrow \mathbb{R}$ defined by

$$
W_{\alpha}(\lambda)=-\left\|\alpha-\left(\lambda p^{j}+(1-\lambda) p^{i}\right)\right\|
$$

Since,

$$
\frac{d W_{\alpha}(\lambda)}{d \lambda}=\frac{1}{W_{\alpha}(\lambda)}\left(\alpha-\left(\lambda p^{j}+(1-\lambda) p^{i}\right)\right) \cdot\left(p^{j}-p^{i}\right)
$$


the function $W_{\alpha}(\lambda)$ is increasing (resp. decreasing) in $\lambda$ whenever $\alpha \cdot\left(p^{j}-p^{i}\right)-$ $\left(\lambda p^{j}+(1-\lambda) p^{i}\right) \cdot\left(p^{j}-p^{i}\right)$ is positive (resp. negative). Therefore, by Assumption $\mathrm{O} 2$, every coalition $C$ of agents of positive measure such that $\alpha \cdot\left(p^{j}-p^{i}\right)>$ $T(p, w(C)) \cdot\left(p^{j}-p^{i}\right)$ for every $\alpha \in C$, will be strictly worse off in joining any (positive measure) coalition of agents deciding to vote for $i$, and, likewise, every agent $\beta \in A$ such that $\beta \cdot\left(p^{j}-p^{i}\right)<T(p, w(C)) \cdot\left(p^{j}-p^{i}\right)$ will be strictly worse off in joining any (non-null) coalition of agents deciding to vote for $j$.

II. Necessity. Suppose not. Let $C$ be a SNE for which the condition does not hold. Fix again $i, j \in M$ with $i \neq j$ and define

$$
D^{j}=C_{j} \cap\left\{\alpha \in A: \alpha \cdot\left(p^{j}-p^{i}\right)<T(p, w(C)) \cdot\left(p^{j}-p^{i}\right)\right\}
$$

Clearly, $D^{j} \in \mathcal{B}$ and, by assumption, $F\left(D^{j}\right)>0$, for some $j=1,2$. Let us suppose, for example, that $F\left(D^{1}\right)>0$. Since $F$ is hyperdiffuse, there exists $\eta>0$ such that $F\left(D_{\eta}^{1}\right)>0$ where the set $D_{\eta}^{1}$ is defined by

$$
D_{\eta}^{1}=D^{1} \cap\left\{\alpha \in A: \alpha \cdot\left(p^{1}-p^{2}\right)+\eta<T(p, w(C)) \cdot\left(p^{1}-p^{2}\right)\right\} .
$$

Using hyperdiffuseness of $F$ again, it is easy to show that there is a coalition $D^{\prime} \subset D_{\eta}^{1}$ of "sufficiently small" measure $\varepsilon=F\left(D^{\prime}\right)>0$. Consider the new coalition $C_{1}^{\prime}=C_{1} \backslash D^{\prime}, C_{2}^{\prime}=C_{2} \cup D^{\prime}$. That is, in the new partition $C^{\prime}$, agents in $D^{\prime}$ have changed their vote from party 1 to party 2 .

Since, $g$ is increasing, we have that $w_{1}\left(C^{\prime}\right)<w_{1}(C)$ as long as $\varepsilon>0$, so $T\left(p, w\left(C^{\prime}\right)\right) \cdot\left(p^{1}-p^{2}\right)<T(p, w(C)) \cdot\left(p^{1}-p^{2}\right)$. By taking $\varepsilon$ small enough, and taking into account that $g$ is also continuous, we may also guarantee that $T(p, w(C)) \cdot\left(p^{1}-p^{2}\right)-\eta<T\left(p, w\left(C^{\prime}\right)\right) \cdot\left(p^{1}-p^{2}\right)$ as well. But now we see that the members of $D^{\prime}$ are strictly better off, so $C$ could not be a SNE.

Finally, note that, since $F$ is hyperdiffuse, the partitioning hyperplane of "indifferent" voters

$$
H=\left\{\alpha \in A: \alpha \cdot\left(p^{1}-p^{2}\right)=T(p, w(C)) \cdot\left(p^{1}-p^{2}\right)\right\}
$$

has zero measure.

Proof of Proposition 1. Fix the policy proposal $p=\left(p_{1}, p_{2}\right)$ and, for each $t \in \mathbb{R}$, let

$$
H(t)=\left\{\alpha \in A: \alpha \cdot\left(p^{1}-p^{2}\right)=t\right\}
$$

denote a hyperplane, normal to the difference of policy vectors. For each $\lambda \in[0,1]$ define

$$
t^{\lambda}=\left(\lambda p^{1}+(1-\lambda) p^{2}\right) \cdot\left(p^{1}-p^{2}\right) .
$$

Notice that $t^{0}=p^{2} \cdot\left(p^{1}-p^{2}\right)$ and $t^{1}=p^{1} \cdot\left(p^{1}-p^{2}\right)$. Thus, $H\left(t^{0}\right)$ (respectively $H\left(t^{1}\right)$ ) corresponds to a hyperplane through $p^{2}$ (respectively $p^{1}$ ). Furthermore, since $t^{\lambda}$ is strictly increasing in $\lambda$, we have that $t^{0}<t^{1}$, whenever $p^{1} \neq p^{2}$. 
Let $C(t) \in \Sigma$ denote the population partition induced by $H(t)$, i.e. $C_{1}(t)=$ $\left\{\alpha \in A: \alpha \cdot\left(p^{1}-p^{2}\right) \geq t\right\}, C_{2}(t)=\left\{\alpha \in A: \alpha \cdot\left(p^{1}-p^{2}\right)<t\right\}$. Define the (continuous) map $h: \mathbb{R} \rightarrow \mathbb{R}$ by

$$
h(t)=h(t ; p)=T(p, w(C(t))) \cdot\left(p^{1}-p^{2}\right) .
$$

For every $t \in \mathbb{R}$, we have that $T(p, w(C(t)))$ belongs to the segment $\left[p^{1}, p^{2}\right]$, because $g^{1} \in[0,1]$. Hence, $t^{0} \leq T(p, w(C(t))) \cdot\left(p^{1}-p^{2}\right) \leq t^{1}$. Thus, $h: \mathbb{R} \rightarrow$ $\left[t^{0}, t^{1}\right]$. Since, $g^{1}$ is strictly increasing, the map $h$ is non-increasing in $t .{ }^{12}$ By the intermediate value theorem, the restriction $h:\left[t^{0}, t^{1}\right] \rightarrow\left[t^{0}, t^{1}\right]$ has a fixed point $t^{*} \in\left[t^{0}, t^{1}\right]$, which satisfies

$$
T\left(p, w\left(C\left(t^{*}\right)\right)\right) \cdot\left(p^{1}-p^{2}\right)=t^{*}
$$

The fixed point $t^{*}$ is unique because $h$ is non-increasing. By Lemma $1, C\left(t^{*}\right)$ corresponds to a unique (up to a zero measure of voters) SNE of this voting game.

Besides insuring existence and uniqueness of the SNE in the party voting game, Proposition 1 restricts the set of the population partitions that may emerge as a voting outcome. In fact, if we ignore deviations by null coalitions, then, given any two distinct policy proposals, the population is partitioned by a hyperplane.

We shall denote the set of all population partitions into two non-empty communities that may be induced by a pluralistic policy profile as $\hat{\Sigma}$. Therefore, in the two-party case $\hat{\Sigma}$ may be taken to be simply the set of all partitions of $A$ by a hyperplane. Each such partition $C \in \hat{\Sigma}$ may be parametrized by the unit normal vector to the partition hyperplane $\pi^{C} \in \mathbb{S}^{n-1}$ (pointing in the direction of $C_{1}$ ) and an intercept $b^{C} \in \mathbb{R}$. As it has been already noted in Caplin and Nalebuff [9], under such a parametrization and ignoring null coalitions, $\hat{\Sigma}$ is identified with an open subset of $\mathbb{S}^{n-1} \times \mathbb{R}$ which is homeomorphic to the whole space $\mathbb{S}^{n-1} \times \mathbb{R} . .^{13}$

Thus, from now on, we will identify the set of population partitions $\hat{\Sigma}$ with the cylinder $\mathbb{S}^{n-1} \times \mathbb{R}$. And, abusing the notation, when there is no confusion, we will not distinguish between population partitions in $\hat{\Sigma}$ and their associated hyperplanes.

In view of the preceding discussion, the voting behavior may be used to define a mapping from the set of all possible pluralistic policy profiles into the set of population partitions $V: \hat{X}^{2} \rightarrow \hat{\Sigma}$, where $\hat{X}^{2}=\left\{p \in X^{2}: p^{1} \neq p^{2}\right\}$, as follows.

For each $p \equiv\left(p^{1}, p^{2}\right) \in \hat{X}^{2}$, we define the induced orientation vector

$$
v_{1}(p)=\frac{p^{1}-p^{2}}{\left\|p^{1}-p^{2}\right\|}
$$

${ }^{12}$ It may actually be constant only if shifting the hyperplane implies a change of decision by a null measure of voters, i.e. if the hyperplane $H(t)$ does not intersect the support of $F$.

${ }^{13}$ As described, $\hat{\Sigma}$ may be identified either with the direct product $\mathbb{S}^{n-1} \times \mathbb{R}$ (which we may view as a cylinder) or with a Mфbius band. The former, however, turns out to be the case, as long as we care about the orientation of the normal vector $\pi$-i.e., the identity of a party adhering to a particular position - which we obviously do here. 
and let $v_{2}(p) \in\left[t^{0}(p), t^{1}(p)\right] \subset \mathbb{R}$ be the unique fixed point of $h(t)$ constructed in the proof of Proposition 1. We define then $V(p)=\left(v_{1}(p), v_{2}(p)\right) \in \mathbb{S}^{n-1} \times$ $\left[t^{0}(p), t^{1}(p)\right]$. Note that other hyperplanes may induce equivalent partitions (i.e. partitions which differ by a null set of agents) as well, as long as the total mass of the population "between" them and the plane $V(p)$ is null.

Lemma 2. Under Assumptions A1, $O 1$ and 02 , the function $V$ is continuous.

Proof. It is immediate that $v_{1}(p)$ is a continuous function on $\hat{X}^{2}$. To see that $v_{2}(p)$ is a continuous function, notice, firstly, that $h(t ; p)$ is continuous in both variables. Recall, that for each $p \in \hat{X}^{2}$ there exists a unique fixed point, $t^{*}(p)$, of $h(t ; p)$. This defines a mapping $p \mapsto t^{*}(p)$ from $\hat{X}^{2}$ to $\mathbb{R}$. By the continuity of Lefschetz's fixed point index (see McLennan [14]), for any open neighborhood $U$ of $t^{*}(p)$ there exists an open neighborhood $W \subset \hat{X}^{2}$ of $p$, such that for each $p^{\prime} \in W$, that this (unique) fixed point $t^{*}\left(p^{\prime}\right)$ must be in $U$. Hence, $v_{2}(p)=t^{*}(p)$ is continuous. ${ }^{14}$

\section{Existence of pluralistic equilibrium}

In general, the problem of existence of pluralistic equilibria is highly non-trivial. In the two-party case, however, we shall provide a rather strong existence result, albeit depending somewhat on the dimension of $A$ and $X$.

In order to do this, we restrict somewhat the class of admissible party statutes. In particular, we would like to avoid policy rules that may depend on the choices made by null coalitions of agents. We will, further, assume that parties would react to "small" (but positive in measure) changes in membership with "small" policy changes. This will be guaranteed by the following two-part assumption.

\section{Assumption P1:}

(i) (irrelevance of null coalitions) For any $C, C^{\prime} \in \Sigma$ which differ by a null coalition of agents, we have that $P(C)=P\left(C^{\prime}\right)$.

(ii) (continuity) $P$ is continuous ${ }^{15}$ when restricted to $\hat{\Sigma}$.

The above assumption allows us to restrict our attention to partitions in $\hat{\Sigma}$ and to insure that the policy proposal profiles induced by partitions in $\hat{\Sigma}$ change continuously with agents' realignment.

At least for membership-based parties, it is not unnatural to assume that, if party membership are on the opposite sides of a hyperplane, the preferred party policies will not be the same.

Assumption P2: (distinct choices) If $C \in \hat{\Sigma}$, then $P^{1}(C) \neq P^{2}(C)$.

14 Note, that existence of the implicit function $t^{*}(p)$ has been shown here without imposing even differentiability of $h$. What we use here might be called a version of the implicit function theorem, stating that, under compactness, the implicit function is continuous whenever it is well-defined, as long as $h(t ; p)$ itself is continuous.

15 As mentioned above, we are identifying the space $\hat{\Sigma}$ of coalitions separated by a hyperplane with $\mathbb{S}^{n-1} \times \mathbb{R}$ endowed with the topology as a subspace of the corresponding Euclidean space. 
In other words, $\mathrm{P} 2$ says that $P(\hat{\Sigma}) \subset \hat{X}^{2}$. Finally, we assume that party statutes do reflect preferences of their members. In particular, we would like to avoid parties making policy proposals relatively unpopular among their own members.

Definition 3. Given a non-null coalition $B \subset A$ and proposals $x, y \in X$ we shall say that $x$ defeats $y$ by a $\delta$-majority, in a (sincere) binary voting (by members of $B)$ if

$$
\frac{F\left(D_{x}\right)}{F(B)} \geq \delta,
$$

where $D_{x}=\{\alpha \in B: u(x ; \alpha) \geq u(y ; \alpha)\}$.

Assumption P3: (minimal primary support) There exists $\eta>0$ such that, for any $C \in \hat{\Sigma}$ for which both parties are non-null and for every $i=1,2$, the proposal $P^{i}(C)$ cannot be defeated by a $(1-\eta)$-majority by any other proposal $x \in X$, in a binary voting by members of $C_{i} .{ }^{16}$

Assumption P3 has a number of significant implications about the policies that can be generated by party statutes. Let $K$ be the convex hull of the support of $F$. Since the support of $F$ is compact, so is $K$. For any $Y \subset \mathbb{R}^{n}$, we let int $Y$ denote the interior of $Y$ and $\partial Y$ its topological boundary. We have the following result.

Lemma 3. Suppose assumptions A1, A2 and P3 hold. Then,

(i) The policy proposal $P^{i}(C) \in \operatorname{int} K$, for every party $i \in M$ and every partition $C \in \hat{\Sigma}$, such that $C_{i}$ is non-null.

(ii) The overall policy outcome $T(P(C), w(C)) \in$ int $K$, for every partition $C \in \hat{\Sigma}$, such that some $C_{i}$ is non-null.

(iii) There exists a compact subset $K_{\eta} \subset \operatorname{int} K$, such that for every $C=\left(C_{1}, C_{2}\right) \in$ $\hat{\Sigma}$ and $i=1,2$, we have that $P^{i}(C) \in K_{\eta}$, whenever $F\left(C_{i}\right) \geq \frac{1}{2}$.

Proof. Part (i) follows from P3. Assume that, for some $i=1,2$, there is $C$ with $F\left(C_{i}\right)>0$ and such that $P^{i}(C)$ is on the boundary of $K$. Choose $q$ so that $\left(q-P^{i}(C)\right) \cdot x \geq 0$ for all $x \in K$ (this is possible, since $K$ is convex). ${ }^{17}$ We may take $q$ arbitrarily close to $P^{i}(C)$. But, this would make the proportion of those party members who prefer $P^{i}(C)$ to $q$, relative to the party membership size, arbitrarily small. $^{18}$

Part (ii) follows immediately from (i), since $K$ is convex.

To see Part (iii), suppose $F\left(C_{i}\right) \geq \frac{1}{2}$ for some $i=1,2$. By assumption P3, $P^{i}(C)$ cannot be defeated by any other policy proposal in an $\eta / 2$-majority binary voting by all agents in $C_{i}$. it follows that $P^{i}(C)$ cannot be defeated by an $\eta / 2$ majority by any other policy proposal in a binary voting by all agents in $A$. Thus, if

16 Of course, given the dimension of the policy space $n$, the minimal support level $\eta$ can always be chosen sufficiently small so that $(1-\eta)$-majority winners actually exist.

17 In other words, $q$ is on the line orthogonal to a supporting hyperplane of the convex hull $K$ at $P^{i}(C)$.

18 We are utilizing here the fact that $F$ is finite and hyperdiffuse over compact support. 
$P^{i}(C)$ were arbitrarily close to the boundary of $K$, then (as in (i)) we may propose an alternative $q$ arbitrarily close to it. ${ }^{19}$ In this way, an arbitrary proportion of agents will prefer $q$ over $P^{i}(C)$, which contradicts Assumption P3.

We provide next an easy characterization of pluralistic equilibria. Consider the function $\phi=\left(\phi_{1}, \phi_{2}\right)=\left(v_{1} \circ P, v_{2} \circ P\right)=V \circ P$. By assumptions $\mathrm{P} 1$ and $\mathrm{P} 2$, the statute profile $P$ maps continuously $\hat{\Sigma}$ into $\hat{X}^{2}$. And, by Lemma $2, V$ is continuous. Hence, $\phi: \hat{\Sigma} \rightarrow \hat{\Sigma}$ is a continuous function, as well.

Lemma 4. If $C^{*} \in \phi\left(C^{*}\right)$, then $\left(P\left(C^{*}\right), C^{*}\right)$ is a pluralistic two-party equilibrium. Furthermore, $C^{\prime} \in \Sigma$ may be an equilibrium partition only if it differs by, at most, a null coalition from some $C^{*} \in \phi\left(C^{*}\right)$.

Proof. The "if" part is trivial from the definition, since $C^{*} \in \phi\left(C^{*}\right)$ means that $C^{*}$ can be supported as a SNE of the voting game between the policy proposals generated by $C^{*}$.

Conversely, consider a pluralistic two-part equilibrium partition $C^{\prime}$. By Lemma 1 , for $C^{\prime}$ to be a SNE of the voting game it may, at most, be different by the vote of a null coalition from some $C^{*} \in \hat{\Sigma}$. Because of the irrelevance of null coalitions (assumption (P1(i)) we know that $P\left(C^{\prime}\right)=P\left(C^{*}\right)$. Therefore, policy proposals induced by $C^{\prime}$ would support in SNE only voters' partitions different by no more than a null coalition from those in $\phi\left(C^{*}\right)$. Hence, the "only if" part of the lemma follows.

We can now state the main result of the paper.

Theorem 1. If assumptions A1-A2, O1-O3, P1-P3 are satisfied, $m=2$ and $n$ is odd, then there exists a pluralistic two-party equilibrium.

The basic idea of the proof is that we may represent pluralistic equilibria as fixed points of a deformation mapping on a compact subset of the cylinder $\mathbb{S}^{n-1} \times \mathbb{R}$, which is homeomorphic to $\mathbb{S}^{n-1} \times[0,1]$. That the set of such fixed points must be non-empty can be shown by applying Lefschetz's fixed point theorem. (For a survey of the mathematical results involved see McLennan [14] and Munkres [15]). Similar ideas have already been used in Caplin and Nalebuff [9] and Gomberg [12].

The proof is broken up into five parts. Parts I and II characterize the relevant subset $\widetilde{\Sigma}$ of $\hat{\Sigma}$; part III defines an auxiliary mapping $\hat{\phi}: \widetilde{\Sigma} \rightarrow \widetilde{\Sigma}$ which coincides with $\phi$ in the interior of $\widetilde{\Sigma}$; part IV shows that $\hat{\phi}$ must have fixed points; and finally, part $\mathrm{V}$ shows that all fixed points of $\hat{\phi}$ are in the interior of the domain and, thus, are also fixed points of $\phi$. By Lemma 4 , these correspond to the required equilibria.

Proof. To avoid possible confusion in the proof, it will be convenient to distinguish between population partitions and the associated hyperplanes. Thus, let us denote by $\Phi(C)=\left(\Phi_{1}(C), \Phi_{2}(C)\right)$ the subsets of the population partition corresponding to the hyperplane $\phi(C)$. We shall also consider the mappings $\pi: \hat{\Sigma} \rightarrow \mathbb{S}^{n-1}$ and $b: \hat{\Sigma} \rightarrow \mathbb{R}$ defined by $\pi(C)=\pi^{C}$ and $b(C)=b^{C}$.

19 Take $q$ on the normal to a supporting hyperplane of $K$ at a boundary point which is "close" to $P^{i}(C)$. 
Part I. We shall prove that there exists $\nu>0$, such that for every equilibrium partition $C$ and every party $i=1,2$ we have that $F\left(C_{i}\right) \geq \nu$. In fact, we will show that there is some $\nu>0$ such that, if $F\left(C_{i}\right)<\nu$ for some party $i=1,2$, then $\Phi_{i}(C) \cap C_{j} \neq \emptyset$, for the other party $j \neq i$.

Indeed, suppose otherwise. Then, we can construct a sequence $\left\{C^{k}\right\}_{k}=$ $\left\{\left(C_{1}^{k}, C_{2}^{k}\right)\right\}_{k}$ of population partitions in $\hat{\Sigma}$, such that $F\left(C_{1}^{k}\right) \rightarrow 0$ and for every integer $k$, we have that $\Phi_{1}\left(C^{k}\right) \subseteq C_{1}^{k}$.

By Lemma 3, for every $C \in \hat{\Sigma}$, we have that $T(P(C), w(C)) \in K$, a compact set. Hence, we may select a subsequence $\left\{C^{k}\right\}_{k}$ (for simplicity, we use the same notation), so that $T\left(P\left(C^{k}\right), w\left(C^{k}\right)\right)$ converges to a point, say $q \in K$.

By Proposition 1, we see that for each $k \in \mathbb{N}$, the point $T\left(P\left(C^{k}\right), w\left(C^{k}\right)\right)$ is in the hyperplane separating $\Phi_{1}\left(C^{k}\right)$ and $\Phi_{2}\left(C^{k}\right)$. And since $\Phi_{1}\left(C^{k}\right) \subseteq C_{1}^{k}$, we must have that $T\left(P\left(C^{k}\right), w\left(C^{k}\right)\right) \in C_{1}^{k}$, for every $k \in \mathbb{N}$. But, $F\left(C_{1}^{k}\right) \rightarrow 0$ implies that any $x \in \operatorname{int} K$ belongs to $C_{2}^{k}$, for some large enough $k$. So, we conclude that $q \in \partial K$.

On the other hand, by assumption $\mathrm{O} 3, g^{1}\left(w\left(C^{k}\right)\right) \rightarrow 0$ and $g^{2}\left(w\left(C^{k}\right)\right) \rightarrow 1$. Hence, $\left\|T\left(P\left(C^{k}\right), w\left(C^{k}\right)\right)-P^{2}\left(C^{k}\right)\right\| \rightarrow 0$. By part (iii) of Lemma 3, we must have that $P^{2}\left(C^{k}\right) \in K_{\eta}$, so $q \in K_{\eta}$ is bounded away from $\partial K$, a contradiction.

Part II. Defining the domain. Take

$$
\widetilde{\Sigma}=\left\{C \in \hat{\Sigma}: \frac{\nu}{2} \leq F\left(C_{i}\right) \leq 1-\frac{\nu}{2}, \quad \text { for } i=1,2\right\} .
$$

Since, the support of $F$ is compact, for each $\pi \in \mathbb{S}^{n-1}$ we may define

$$
\underline{b}(\pi)=\inf \left\{b^{C}: \pi^{C}=\pi, C \in \widetilde{\Sigma}\right\}
$$

and

$$
\bar{b}(\pi)=\sup \left\{b^{C}: \pi^{C}=\pi, C \in \widetilde{\Sigma}\right\} .
$$

Thus, for every $C \in \widetilde{\Sigma}$ with the first coordinate $\pi^{C} \in \mathbb{S}^{n-1}$, the intercept $b^{C}$ belongs to the closed interval $\left[\underline{b}\left(\pi^{C}\right), \bar{b}\left(\pi^{C}\right)\right]$.

It follows from hyperdiffuseness of $F$ that, both $\underline{b}(\pi)$ and $\bar{b}(\pi)$, are continuous functions of $\pi \in \mathbb{S}^{n-1}$. This implies that $\widetilde{\Sigma}$ is homeomorphic to $\mathbb{S}^{n-1} \times[0,1]$, a compact cylinder. Since, $[0,1]$ is contractible, $\mathbb{S}^{n-1}$ is deformation retract of $\widetilde{\Sigma}$.

Part III. The function $\phi$ may map some partitions from $\widetilde{\Sigma}$ into $\Sigma \backslash \widetilde{\Sigma}$. We shall, therefore, construct a continuous function $\hat{\phi}: \widetilde{\Sigma} \rightarrow \widetilde{\Sigma}$ as follows. The mapping $\hat{\phi}=\left(\hat{\phi}_{1}, \hat{\phi}_{2}\right)$ is defined by taking $\hat{\phi} \equiv \phi$, when $\phi(C) \in \widetilde{\Sigma}$; but, by replacing each $\phi(C)$ with

$$
\hat{\phi}(C)=\left\{\begin{array}{l}
\left(\phi_{1}(C), \underline{b}\left(\phi_{1}(C)\right)\right) \text { if } \phi_{2}(C)<\underline{b}\left(\phi_{1}(C)\right) \\
\left(\phi_{1}(C), \bar{b}\left(\phi_{1}(C)\right) \text { if } \phi_{2}(C)>\bar{b}\left(\phi_{1}(C)\right)\right.
\end{array}\right.
$$

That is, we let $\hat{\phi_{1}}=\phi_{1}$ and $\hat{\phi_{2}}=\max \left\{\underline{b} \circ \phi_{1}, \min \left\{\phi_{2}, \bar{b} \circ \phi_{1}\right\}\right\}$. Thus, $\hat{\phi}$ inherits the continuity from $\phi$. 
Part IV. Now we prove that the set $\mathcal{F}(\hat{\phi})$ of fixed points of $\hat{\phi}$ is non-empty.

By Lemma 3, we see that $P^{i}(C) \in \operatorname{int} K \cap C_{i}$ for every $i=1,2$. Hence, the inner product $\pi^{C} \cdot\left(P^{1}(C)-P^{2}(C)\right)>0$. Therefore,

$$
\phi_{1}(C)=\frac{P^{1}(C)-P^{2}(C)}{\left\|P^{1}(C)-P^{2}(C)\right\|} \neq-\pi^{C} .
$$

And we see that $\hat{\phi}_{1}(C)=\phi_{1}(C) \neq-\pi^{C}$, for every $C \in \widetilde{\Sigma}$.

We can chose $\nu$ sufficiently small and a point $\xi$ such that all partitions by hyperplanes through it belong to $\widetilde{\Sigma}$. Let $\Psi$ denote this set. Clearly, $\Psi$ is homeomorphic to $\mathbb{S}^{n-1}$ and may be parametrized by $\pi^{C}$. Furthermore, one may easily construct a retraction $r$ from $\widetilde{\Sigma}$ onto $\Psi=\mathbb{S}^{n-1}$. We can do this, for instance, by identifying $\Psi$ with $\mathbb{S}^{n-1} \times\{0\} \subset \widetilde{\Sigma}$ and $\widetilde{\Sigma}$ with $\mathbb{S}^{n-1} \times[0,1]$. Under this identification, the retraction map is $r(\pi, b)=(\pi, 0)$. Consequently, we regard $\phi_{1}$ as a mapping $\phi_{1}: \widetilde{\Sigma} \rightarrow \Psi$.

Consider now the map $i \circ \phi_{1} \circ r: \widetilde{\Sigma} \rightarrow \widetilde{\Sigma}$, where $i$ is the inclusion map from $\Psi=\mathbb{S}^{n-1} \times\{0\}$ into $\widetilde{\Sigma}=\mathbb{S}^{n-1} \times[0,1]$. Using the above coordinates, we see that $i\left(\phi_{1}(r(\pi, b))\right)=\left(\phi_{1}(\pi, 0), 0\right)$. Since, $\phi_{1}(C) \neq-\pi^{C}$ for every $C \in \Psi$, we have that $i \circ \phi_{1} \circ r$ is homotopic to the identity map. For instance, a homotopy map $H: \widetilde{\Sigma} \times[0,1] \rightarrow \widetilde{\Sigma}$, between $i \circ \phi_{1} \circ r$ and the identity, is given by

$$
H((\pi, b), t)=\left(\frac{t \pi+(1-t) \phi_{1}(\pi, 0)}{\left\|t \pi+(1-t) \phi_{1}(\pi, 0)\right\|}, t b\right)
$$

Therefore, the Lefschetz number, $\Lambda\left(i \circ \phi_{1} \circ r\right)=\Lambda$ (identity) and the latter coincides with $\chi(\widetilde{\Sigma})$, the Euler characteristic of the space $\widetilde{\Sigma}$ (Munkres [15]). But, since $\widetilde{\Sigma}$ is homeomorphic to the sphere $\mathbb{S}^{n-1} \times[0,1]$, its Euler characteristic is $\chi(\widetilde{\Sigma})=\chi\left(\mathbb{S}^{n-1}\right)=2$, for odd $n$.

In addition, there is a homotopy $\widetilde{H}: \widetilde{\Sigma} \times[0,1] \rightarrow \widetilde{\Sigma}$, between the mappings $\hat{\phi}$ and $i \circ \phi_{1} \circ r$, given by

$$
\widetilde{H}((\pi, b), t)=\left(\phi_{1}(\pi, b t), t \hat{\phi}_{2}(\pi, b t)\right) .
$$

Hence, $\Lambda(\hat{\phi})=\Lambda\left(i \circ \phi_{1} \circ r\right)=2$, does not vanish. By the Lefschetz fixed point theorem (McLennan [14]), $\mathcal{F}(\hat{\phi}) \neq \emptyset$.

Part V. We show, finally, that $\mathcal{F}(\hat{\phi}) \subset$ int $(\Sigma)$.

Suppose otherwise. Then, we can find a partition $C^{*} \in \partial \widetilde{\Sigma}$ such that $\hat{\phi}\left(C^{*}\right)=$ $C^{*}$. Hence, $F\left(C_{i}^{*}\right)=\nu / 2$ for some $i=1,2$. Say, $F\left(C_{1}^{*}\right)=\nu / 2$. But then, we have that $F\left(C_{1}^{*}\right)<\nu$ and $\Phi_{1}(C) \cap C_{2}^{*}=C_{1}^{*} \cap C_{2}^{*}=\emptyset$, which contradicts the claim in part I.

To finish the proof of the Theorem, note that $\hat{\phi}(C)=\phi(C)$ as long as $C \notin \partial \widetilde{\Sigma}$. Thus, Part V implies that $\mathcal{F}(\hat{\phi})=\mathcal{F}(\phi)$, and we conclude that $\mathcal{F}(\phi) \neq \emptyset$. 
The existence result obtained above for the odd dimensional policy spaces is quite general, in that it imposes relatively few restrictions on the internal policy rules of the parties. One may argue that, since we are unlikely to observe precisely political decision processes, both within parties and in the society as a whole, a model which depends in a discontinuos way on changes in the specifications of these processes is not likely to result in reliable predictions. Nevertheless, it is possible to guarantee the robustness of the equilibria implied by Theorem 1 to small changes in party statutes. ${ }^{20}$

Unfortunately, when the dimension of the policy space is even, we may only achieve more limited results. The following example shows an even-dimensional model satisfying all our assumptions and for which there is no Pluralistic Equilibrium. The construction of this example is closely related to the well known fact that, there exists a continuous non vanishing vector field on the sphere $\mathbb{S}^{k}$, if and only if $k$ is odd.

Example 1. There are two political parties, $M=\{1,2\}$. For simplicity, we assume that the policy space $X=\left\{x \in \mathbb{R}^{2 n}:\|x-z\| \leq 1\right\}$ is a closed disc of dimension $2 n$ with center $z \in \mathbb{R}^{2 n}$. The boundary of $X$ is $\mathbb{S}^{2 n-1}$ an odd dimensional sphere. For this example, one only needs that $X$ is a compact, convex set whose boundary is homeomorphic to the sphere $\mathbb{S}^{2 n-1}$.

Let $Y$ be a continuous non-vanishing vector field on $\mathbb{S}^{2 n-1}$. We define the policy rule as follows. Let $C \in \hat{\Sigma}$ be a population partition and let $H(C)$ be the hyperplane that separates $C_{1}$ and $C_{2}$. The intersection of $H(C)$ with $X$ is a $2 n-1$ dimensional closed disk with center $Z(C)$. Let $r(C)$ be the line through $Z(C)$ orthogonal to $H(C)$. The line $r(C)$ intersects the boundary of $X$ in two opposite points $e_{i} \in C_{i}, i=1,2$. Let $d^{i}(C) \in C_{i}$, for $i=1,2$ be the midpoint on $r(C)$ between $Z(C)$ and $e_{i}$. For each $C \in \hat{\Sigma}$, the points $d^{i}(C)$ are in the interior of $C_{i}$.

We construct now a continuous map $g: \hat{\Sigma} \rightarrow R_{++}$such that, for every partition $C=\left(C_{1}, C_{2}\right)$ and for each coalition $i=1,2$, the points $d^{i}(C) \pm g(C) Y\left(e_{i}\right)$ are in the interior of $C_{i}$. We let now $p^{1}(C)=d^{1}(C)+g(C) Y\left(e_{1}\right)$ and $p^{2}(C)=$ $d^{2}(C)-g(C) Y\left(e_{1}\right)$. One checks easily that the functions $p^{1}(C)$ and $p^{2}(C)$ are continuous.

By construction, for any given partition $C$, the segment $\left[p^{1}(C), p^{2}(C)\right]$ is not perpendicular to the hyperplane $H(C)$. Thus, no pair of the form $(P(C), C)$ can be a pluralistic multi-party equilibrium.

\section{The mean voter rule}

While the example in the previous section highlights the difficulty of establishing general existence results when there is an even number of policy dimensions, we may still be able to obtain more limited results for some interesting classes of policy rules. As an important example we shall show that, when policies are twodimensional and each party chooses for its proposal the ideal point of its mean member, a two-party equilibrium must exist.

\footnotetext{
20 The details of this result which, for the sake of conciseness have been removed from the present version of the paper, are available upon request from the authors
} 
Let us assume that the individual distribution on $A$ is given by the non-atomic measure $F$ with a density $f(x)$ and, for simplicity, identify the set of agents with the set of policies, i.e., $X=A \subset \mathbb{R}^{2}$. As above, there are two parties and the weights are given by the total population in each coalition

$$
\omega(C)=\left(\omega^{1}\left(C_{1}\right), \omega^{2}\left(C_{2}\right)\right)=\left(\int_{C_{1}} f(x) d x, \int_{C_{2}} f(x) d x\right)
$$

But now, we specify that the party policy choice of each coalition $i=1,2$ is its center of gravity

$$
\begin{aligned}
P^{i}\left(C_{i}\right) & =\frac{1}{\omega^{i}\left(C_{i}\right)} \int_{C_{i}} x f(x) d x \\
& =\frac{1}{\omega^{i}\left(C_{i}\right)}\left(\int_{C_{i}} x_{1} f(x) d x, \int_{C_{i}} x_{2} f(x) d x\right)
\end{aligned}
$$

where $x=\left(x_{1}, x_{2}\right) \in X$ and we have assumed that $\omega^{i}\left(C_{i}\right) \neq 0$. To avoid cumbersome notation, let us write $p_{i}=P^{i}\left(C_{i}\right), \omega^{i}=\omega^{i}\left(C_{i}\right)$, for $i=1,2$, when there is no possibility of confusion.

The outcome function $T(p, \omega)=\omega^{1}\left(C_{1}\right) p^{1}+\omega^{2}\left(C_{2}\right) p^{2}=\int_{X} x f(x) d x$ therefore results in a policy outcome which is independent of the partition. After a translation, we may (and will) assume that $T(p, \omega)=(0,0) \in X$.

With this convention, $p^{1}$ and $p^{2}$ are colineal, pointing in opposite directions. In general, $p^{1}-T(p, \omega)$ and $p^{2}-T(p, \omega)$ are colineal, pointing in opposite directions.

Proposition 2. Suppose that $n=2$ and the policies chosen by the parties are given by the rule in Equation 5.2. Then, there is a partition $C=\left(C_{1}, C_{2}\right)$ such that $(P(C), C)$ is a pluralistic equilibrium.

Proof. By Proposition 1, the Strong Nash Equilibria correspond to coalitions $C_{1}$ and $C_{2}=X \backslash C_{1}$ which are separated by a straight line $H(C)$ containing the point $T(p, \omega)=(0,0)$. We identify the unit vector, say $q$, orthogonal to the line $H(C)$ with the partition $C=\left\{C_{1}, C_{2}\right\}$ so,

$$
C_{1}=\{x \in X: q \cdot x \leq 0\}, C_{2}=\{x \in X: q \cdot x>0\} .
$$

Since $X$ is convex, by changing to polar coordinates we may write

$$
X=\{(r \cos \theta, r \sin \theta): 0 \leq r \leq r(\theta), 0 \leq \theta<2 \pi\}
$$

for some function $r:[0,2 \pi) \rightarrow \mathbb{R}_{+}^{n}$. And we see that, the partition induced by the vector $q=(\cos \alpha, \sin \alpha)$ is then,

$$
\begin{gathered}
C_{1}=\left\{(r \cos \theta, r \sin \theta): 0<r \leq r(\theta), \alpha-\frac{\pi}{2} \leq \theta \leq \alpha+\frac{\pi}{2}\right\} \\
C_{2}=\left\{(r \cos \theta, r \sin \theta): 0 \leq r \leq r(\theta), \alpha+\frac{\pi}{2}<\theta<\alpha+\frac{3 \pi}{2}\right\}
\end{gathered}
$$


We remark that $q=(\cos \alpha, \sin \alpha) \equiv C=\left\{C_{1}, C_{2}\right\}$ is a pluralistic equilibrium if and only if $q$ is colineal with the vectors $p^{1}$ and $p^{2}$, that is if and only if $p^{1}$ and $p^{2}$ are orthogonal to the half-line $\left\{r(\cos \theta, \sin \theta): 0 \leq r<\infty, \theta=\alpha+\frac{\pi}{2}\right\}=\{r(-\sin \alpha, \cos \alpha): 0 \leq r<\infty\}$.

Thus, the problem is reduced to show that we can find $\alpha \in[0,2 \pi)$ such that $G(\alpha)=0$, where

$$
G(\alpha)=-\sin \alpha \int_{C_{1}} x_{1} f(x) d x+\cos \alpha \int_{C_{1}} x_{2} f(x) d x
$$

Changing to polar coordinates, and using Fubini's Theorem, this is the same as

$$
\begin{aligned}
& -\int_{\alpha+\frac{\pi}{2}}^{\alpha+\frac{3 \pi}{2}} \int_{0}^{r(\theta)} r^{2} \sin \alpha \cos \theta f(r, \theta) d r d \theta \\
& +\int_{\alpha+\frac{\pi}{2}}^{\alpha+\frac{3 \pi}{2}} \int_{0}^{r(\theta)} r^{2} \cos \alpha \sin \theta f(r, \theta) d r d \theta \\
= & \int_{\alpha+\frac{\pi}{2}}^{\alpha+\frac{3 \pi}{2}} \int_{0}^{r(\theta)} \sin (\theta-\alpha) r^{2} f(r, \theta) d r d \theta \\
= & \int_{\alpha+\frac{\pi}{2}}^{\alpha+\frac{3 \pi}{2}} \sin (\theta-\alpha) g(\theta) d \theta
\end{aligned}
$$

with

$$
g(\theta)=\int_{0}^{r(\theta)} r^{2} f(r, \theta) d r
$$

Note that $g(\theta+2 \pi)=g(\theta)$. Making the change of variable $\theta=t+\alpha$, we see that $G(\alpha)$ equals

$$
\int_{\frac{\pi}{2}}^{\frac{3 \pi}{2}} g(t+\alpha) \sin t d t
$$

Integrating now the function $G(\alpha)$ and using again Fubini's Theorem, we obtain

$$
\int_{0}^{2 \pi} G(\alpha) d \alpha=\int_{\frac{\pi}{2}}^{\frac{3 \pi}{2}} \sin t\left(\int_{0}^{2 \pi} g(t+\alpha) d \alpha\right) d t
$$

But

$$
\int_{0}^{2 \pi} g(t+\alpha) d \alpha=\int_{0}^{2 \pi} g(\alpha) d \alpha=A
$$

is independent of $t$, because $g(\theta+2 \pi)=g(\theta)$. Hence,

$$
\int_{0}^{2 \pi} G(\alpha) d \alpha=A \int_{\frac{\pi}{2}}^{\frac{3 \pi}{2}} \sin t d t=0
$$

and, by continuity of $G$, there must be some $\alpha \in[0,2 \pi)$ such that $G(\alpha)=0$. 
This result is, admittedly, more limited than the one obtained in the previous section for the odd-dimensional case. Nonetheless, in the present setting the meanvoter rule has some interest. Since, Caplin and Nalebuff [9] have shown that, under some conditions on the distribution of individuals, the mean-voter proposal cannot be defeated by any other alternative by a qualified majority in a binary vote. Also, Grofman and Feld [10] have proved that, for $n=2$, the winner under the mean voter rule coincides with the winning proposal under the (generalized) Borda count.

\section{References}

1. Aldrich, J. H.: A Downsian spatial model of party activism. American Political Science Review 77, 974-990 (1983)

2. Alesina, A.: Credibility and policy convergence in a two-party system with rational voters. American Economic Review 78, 796-806 (1988)

3. Alesina, A., Rosenthal, H.: Partisan politics, divided government, and the economy. Cambridge, MA: Cambridge University Press (1996)

4. Alesina, A., Rosenthal, H.: A theory of divided government. Econometrica 64, 1311-1341 (1997)

5. Aumann, R. J.: Acceptable points in general cooperative n-person games. In: Tucker, A. W., Luce, R. D. (eds.) Contributions to the theory of games, Vol. IV, pp. 287-324 (Annals of Mathematics Studies, 40). Princeton: Princeton University Press 1959

6. Baron, D. P.: Government formation and endogenous parties. American Political Science Review 87, 34-47 (1993)

7. Besley, T., Coate, S.: An economic model of representative democracy. Quarterly Journal of Economics, Febr., 85-114 (1997)

8. Caplin, A., Nalebuff, B.: Aggregation and social choice: A mean voter theorem. Econometrica 59, 1-24 (1991)

9. Caplin, A., Nalebuff, B.: Competition among institutions. Journal of Economic Theory 72, 306-342 (1997)

10. Feld, S., Grofman, B.: The Borda Count in $n$-dimensional issue space. Public Choice 59, 167-176 (1988)

11. Gerber, A., Ortuño-Ortín, I.: Political compromise and endogenous formation of coalitions. Social Choice and Welfare 15, 445-454 (1998)

12. Gomberg, A.: Equilibrium in a multi-jurisdiction model. Journal of Economic Theory (forthcoming)

13. Grossman, G., Helpman, E.: Electoral competition and special interest politics. Review of Economic Studies 63, 265-286 (1996)

14. McLennan, A.: Selected topics in the theory of fixed points. University of Minnesota Center for Economic Research Discussion paper No. 251 (1989)

15. Munkres, J. R.: Elements of algebraic topology. New York: Addison-Wesley 1984

16. Ortuño-Ortín, I.: A spatial model of political competition and proportional representation. Social Choice and Welfare 14, 427-438 (1997)

17. Ortuño-Ortín, I., Roemer, J. E.: Endogenous party formation and the effect of income distribution on policy. WP-AD 2000-06, University of Alicante, Spain (1998)

18. Osborne, M.: Spatial models of political competition under plurality rule: A survey of some explanations of the number of candidates and the positions they take. Canadian Journal of Economics 28, 261-301 (1995)

19. Osborne, M., Slivinski, A.: A model of political competition with citizen-candidates. Quarterly Journal of Economics CXI, 65-96 (1996)

20. Osborne, M., Tourky, R.: Party formation in collective decision-making. Research Paper No. 844, University of Melbourne (2002)

21. Poutvaara, P.: Party platforms with endogenous party membership. Public Choice 117, 77-98 (2003)

22. Riviere, A.: Citizen candidacy, party formation and Duverger's law. Discussion Papers in Economics - dpe00/1 - 2000, University of London (1999) 
23. Roemer, J. E.: The democratic political economy of progressive taxation. Econometrica 67, 1-20 (1999)

24. Roemer, J. E.: Political competition. Cambridge, MA: Harvard University Press 2000

25. Wittman, D.: Parties as utility maximizers. American Political Science Review 67, 490-498 (1973) 